\title{
Schräger Tipp für einen gesunden Rücken
}

\author{
Chefredakteur Dr. med. Dirk Einecke
}

\section{TODESURSACHEN-TRENDS}

\section{Tabak und HIV auf der Überholspur}

Todesfälle durch Herzkrankheiten, Krebs und Diabetes werden in den nächsten Jahren weiter zunehmen, während die meisten Infektionskrankheiten als To-



desursache zurückgehen werden. Mit einer Ausnahme: Die Zahl der AIDS-Toten wird weiter ansteigen. In 25 Jahren wird die HIV-Infektion weltweit die dritthäufigste Todesursache sein. Krankheiten, die durch Rauchen begünstigt werden, werden ebenfalls weiter zunehmen. Die Weltgesundheitsorganisation (WHO) prognostiziert, dass im Jahr 2030 8,3 Mio. Menschen wegen ihres Tabakkonsums, 6,5 Mio. an AIDS und 2,1 Mio. an Verkehrsunfällen sterben werden. Bis 2015 wird der Zigarettenkonsum bereits für jeden zehnten sein.

Quelle: Updated projections of disease, 2002-2030, WHO Todesfall verantwortlich global mortality and burden of

\section{NUR VÖLLIGE ABSTINENZ SCHÜTZT}

\section{Nicht-Rauchen light bringt nichts}

Raucher, die ihren Zigarettenkonsum nur drosseln, aber nicht völlig aufgeben, sind gegenüber denjenigen, die unverändert weiterrauchen, gesundheitlich nicht im Vorteil. Allein eine strikte Tabakabstinenz verringert das Risiko für kardiovaskuläre oder maligne Erkrankungen, stellte eine norwegische Langzeitstudie mit 51000 Teilnehmern fest. Ein Teil der Probanden reduzierte ihren Tabakkonsum von initial über 15 auf weniger als acht Zigaretten pro Tag, eine Gruppe rauchte unverändert weiter und die dritte Gruppe hörte ganz zu Rauchen auf. Die Todesrate und Häufigkeit von Tabak-assoziierten Krankheiten unterschieden sich nach 25 Jahren in den ersten beiden Gruppen nicht. In der dritten Gruppe sank das Risiko jedoch um die Hälfte.

Tobacco Control, Vol 15, 2006, 472

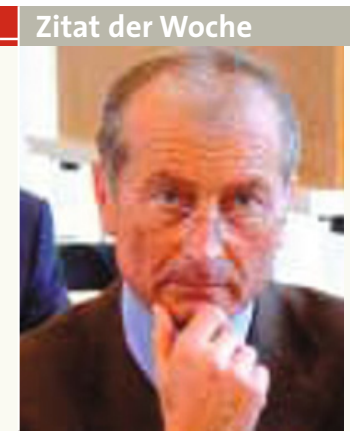

„Ich empfehle dem KVB-Vorstand, bei der nächsten Einladung eine Feldflasche und ein Butterbrot mitzunehmen, damit ihm nicht vorgeworfen werden kann, sich auf Pharma-Kosten ernährt zu haben."

Dr. Wolf Neher, Geretsried, auf der Vertreterversammlung am 25.11.2006 in München, nachdem der Hausärzteverband dem KVB-Vorsitzenden Dr. Axel Munte vorgehalten hatte, an einer Pharma-gesponsorten Veranstaltung teilgenommen zu haben.

\section{RÜCKENSCHONENDER RAT}

\section{Sitz nicht gerade!}

Wer viele Stunden am Tag sitzend verbringt, sollte eine Position einnehmen, die den Rücken möglichst wenig belastet. Schottische Ärzte haben herausgefunden, dass das am ehesten gelingt, wenn man sich entspannt in einem $135^{\circ}$-Winkel im Stuhl zurücklehnt. Bei einer ganz aufrechten ebenso wie einer vornüber gebeugten Sitzhaltung wird mehr Druck auf die Bandscheiben ausgeübt, wie MRT-Untersuchungen bei 22 gesunden Probanden ergaben.

W. A. Bashir, Kongress der Radiological Society of North America, Chicago, 27. November 2006

\section{STRUKTURELLE RESERVEKAPAZITÄT}

\section{Dickkopf schützt vor Demenz}

Je kleiner der Kopf, desto größer ist das Demenzrisiko. Eine Querschnittsstudie bei 442 Nonnen im Alter über 65 Jahren hat ergeben, dass Frauen, deren Kopfumfang unter dem Durchschnitt lag, doppelt so häufig unter einer Demenz litten wie ihre Ordensschwestern mit größeren Köpfen. Offenbar dient das Mehr an Hirnmasse als Reserve gegenüber dem fortschreitenden neuronalen Abbau. Als weitere strukturelle Schutzfaktoren haben sich Geburtsgewicht, postnatales Hirnwachstum und Körpergröße erwiesen. Dr. H. Bickel auf dem DGPPN-Kongress, Berlin 22.-25.11.2006

\section{INFLUENZA-IMPFUNG FÜRS PERSONAL}

\section{Lebensrettende Maßnahme für Pflegeheimbewohner}

Mitarbeiter von Pflegeheimen sollten gegen Grippe geimpft sein. Dadurch lassen sich die Erkrankungsrate und die Todesfälle unter Heimbewohnern in Zeiten mit mäßiger Influenzaaktivität signifikant reduzieren. Der Schutzeffekt besteht auch dann, wenn die Heimbewohner zum größten Teil selbst geimpft sind. Denn gerade bei älteren Menschen ist wegen der verminderten Immunantwort der Impfschutz gegen Influenza oft unvollständig. Brit Med J 30. Nov. 2006 